\title{
All-metal 3-D Frequency Selective Surface with Versatile Dual-Band Polarization Conversion
}

\author{
C. Molero, Member, IEEE, E. Menargues and M. García-Vigueras, Member, IEEE,
}

\begin{abstract}
A dual-band periodic structure is here proposed, which allows for versatile frequency selectivity and polarization conversion functionality. The structure is a monolithic fullmetal piece, and it is built from the arrangement of unit-cells consisting of waveguide sections at cutoff loaded with compound perforations. The topology of such unit-cells enables the efficient and independent interaction with vertical and horizontal electric field components illuminating the periodic structure. A circuit model is proposed to characterize the cells and understand their behaviour. Three different examples of cells are designed to illustrate the structure versatile functionality. One of these examples is manufactured, consisting of a dual-band structure that converts linear polarization into circular with orthogonal senses of rotation at each band. The experimental results are in good agreement with the theoretical predictions, thus validating the underlying operation mechanism and its practical feasibility.
\end{abstract}

Index Terms-3-D periodic structures, polarizers, equivalent circuit

\section{INTRODUCTION}

$\mathbf{T}$ HREE-dimensional frequency selective surfaces (3-D FSSs) consist typically on the periodic arrangement of unit-cells that are non-uniform along the propagation direction, in contrast to their two-dimensional (2-D) counterparts. This important aspect allows for additional degrees of freedom in the conception of radio-frequency (RF) devices with enhanced performance. Multiple recent examples illustrate the potential of 3-D FSSs operating as absorbers and rasorbers [1], bandstop or bandpass filters [2] as well as polarizers [3].

The discovery of metamaterials put 3-D periodic structures in the RF academic spotlight at the beginning of this century [4]. As illustrated in [5], the periodic arrangement of split ring resonators oriented along the three spacial directions allows to synthesize an artificial material having both negative effective permittivity and permeability. More recently, advanced manufacturing techniques and 3D-printing have boosted again the exploration of 3-D structures.

The need to move towards 3-D topologies is explained in [1], [6], and illustrated with the conception of a wideband

Manuscript received 31 - 07 - 2019; revised 24 - 11- 2019; accepted 02 02 - 2020. This publication has been supported by Rennes Métropole under Grant AIS 17C0481, the Brittany region under Contract SAD 2016 9637, the European Union through the European Regional Development Fund (ERDF), and by Ministry of Higher Education and Research, Brittany and Rennes Métropole, through the CPER Project SOPHIE / STIC and Ondes.

Carlos Molero and María García-Vigueras are with the Institute d'Electronique et Télécommunications de Rennes, UMR CNRS 6164, INSA de Rennes, Rennes (e-mail: carlos.molero-jimenez@insa-rennes.fr, maria.garcia-vigueras@insa-rennes.fr).

Esteban Menargues is with Swissto12, Ecublens, Switzerland (e-mail: e.menargues@swissto12.ch).

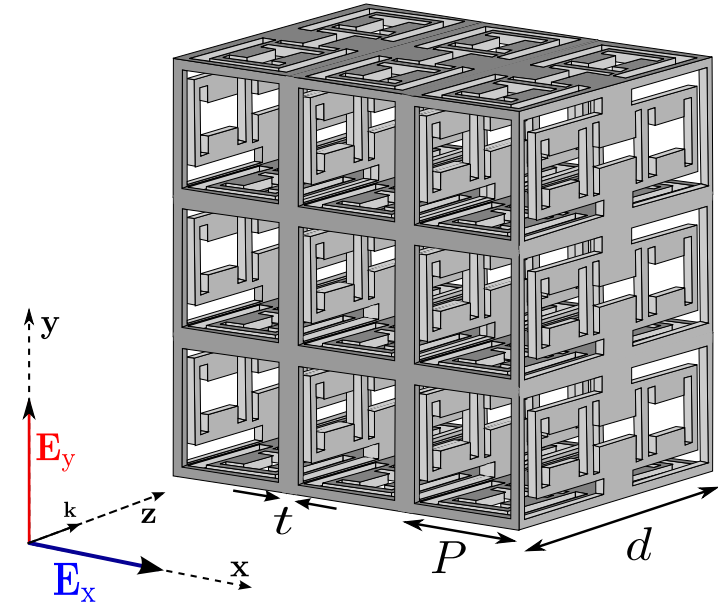

Fig. 1. Proposed all-metal FSS based on 3-D structure. The excitation is considered to be a plane wave impinging normally. The corresponding electric field is decomposed into vertical and horizontal components, $\mathbf{E}_{\mathrm{y}}$ and $\mathbf{E}_{\mathrm{x}}$ respectively. The unit-cell periodicity is $P$, its length along the $z$ axis is $d$ and the thickness of the metallic walls is $t$.

absorber. In such an example, the insertion of lumped elements along the propagation direction (exploiting the third dimension) of printed-circuit cells allows the device performance to be enhanced and to simplify the design process. A different configuration of absorber is proposed in [7] based on 3-D printed resonant cells built from circular slots.

The conception of stop-band FSSs employing 3-D cells is also addressed in the literature, either based on printed-circuit board (PCB) cells [8], [9] or 3-D printed elements [10], [11]. The synthesis of passband filtering is more diverse. Band widening is attained in [2], [12] by using cells built from dielectric sections pierced by bifilar lines. Wideband behaviour and angular robustness has been demonstrated by using interleaved metallic architectures [13], polymethylmethacrylate lattices [14], or typical PCB cells [15]. The monolithic passband structures proposed in [16]-[18] consist of arrays of square metallic waveguides. These topologies independently interact with orthogonal field components, and they inspire the conception of the structure considered in this paper.

Linear-to-circular converters constitute another application that can profit from 3-D periodic structures. So far, research efforts have mainly concentrated on the development of PCB polarizing screens. Recent proposals consist on PCB screens with wideband operation [19] or multiband behaviour [20], [21]. Very few examples consider cells with 3-D topologies, some of them are discussed next. In [22], chiral geometries are used to control the phase delay induced between illu- 
minating orthogonal field components. Wideband conversion is attained in [23] based on dielectric grooved rods. Cells based on metallic helices are theoretically studied in [24], though no experimental results are provided. More recently, some of the authors of this paper have proposed a concept to conceive polarizing architectures based on monolithic metallic structures [3], [25]. Again, this remains a theoretical study and only single-band operation is provided. Dual-band polarizers allowing orthogonal senses of circular polarization (CP) in each band are attracting much interest in the present decade due to their application in K/Ka-band satcom antennas [26][31]. While [26] and [27] remain theoretical proposals, specific prototypes are reported in [28]-[31] that are built from standard PCB manufacturing. The thickness of such PCB designs is low, but they rely on the use of dielectric materials as support, which imply significant losses at millimetre waves and are commonly avoided in space application. The use of equivalent circuits is employed in some cases to explain the polarizer operation.

In this paper, a versatile 3-D FSS unit-cell is proposed operating in dual band. Inspired from [16], the cell consists on a waveguide operating below cutoff perforated with slots in its lateral walls. No circuit model has yet been proposed to characterize such a structure, which is needed to accurately understand its behaviour and to fully profit from its potential. Circuit models are well established for planar 2-D geometries [32], [33], but they are not yet formalized for 3-D FSS scenarios. Theoretical models based on circuital assumptions or mathematical deductions can be found in some of the references cited above, such as [1], [8], [34]. Quasi-analytical equivalent circuits have been recently proposed by some of the authors in [3], [25], providing good accuracy and valuable physical understanding. Such models are here extended to characterize the proposed dual-band structure. In particular, the models allow to understand the versatility of the unit-cell and its capability to alter the polarization of the impinging wave independently at each band.

Finally, in this contribution we address the specific design of all-metal dual-band polarizers inducing orthogonal sense of $\mathrm{CP}$ at each band. A prototype operating around $10 \mathrm{GHz}$ and $15 \mathrm{GHz}$ is designed, manufactured and measured. The good agreement between the experimental results and the theoretical predictions not only validates the proposed concept, but also proves for the first time, the feasibility to conceive all-metal polarizing structures. The avoidance of dielectric materials or other supports adds important originality to this contribution, and represents the main advantage of the present polarizer. The concept underlying polarization conversion in a monolithic and all-metal 3-D structure could become a promising starting point for the development of low-loss external polarizers for operation in space or other harsh environments, where dielectric materials are avoided as much as possible. Many illustrative examples can be found in the literature justifying the interest in all-metal radiating structures (see for example [35]-[37] targeting at band X , L or K). These works reveal that, in addition to avoiding undesired electromagnetic phenomena (such as ESD or PIM), all-metal architectures allow to prevent the tolerances that are inherent to dielectric materials (mainly related to the permittivity). In this case, the agreement between the performance of a theoretical model and a prototyped device depends only on the simulation accuracy and the manufacturing tolerances.

This paper is organized as follows. Section II describes the structure of the proposed unit-cell and the topology of its equivalent circuit. Section III illustrates the versatility of the cell by means of three theoretical examples. Finally, Section IV presents the experimental validation of the manufactured prototype. The agreement between measured and theoretical results is discussed, together with the details about the employed measurement setup.

\section{STRUCTURE DESCRIPTION AND MODELLING}

This section presents the 3-D topology and the concept that allows for transmission of dual-polarized electromagnetic waves impinging normally, as well as independent phase manipulation, through an all-metal monolithic structure. The physical principle underlying such operation is supported through the modelling with an equivalent circuit.

\section{A. Unit-cell description}

A 3x3 sample of the proposed FSS is illustrated in Fig. 1. The structure can either be illuminated by a vertical electric field $\mathbf{E}_{\mathrm{y}}$, an horizontal one $\mathbf{E}_{\mathrm{x}}$, or a combination of both. The unit-cells in the FSS are built from portions of length $d$ of square waveguides whose section of length $P-t$ is smaller than half a wavelength at the highest frequency of operation. The sketch of a single cell is illustrated in Fig. 2(a), where the slots perforated at its walls can be clearly observed. Two type of perforations can be distinguished: those having the shape of a folded slot (located around the limits of the walls), and H-shaped apertures (occupying the center region). This particular configuration allows long folded slots to surround the H-shaped ones, in order to efficiently occupy the surface of the walls. In a single wall, two identical folded slots with opposite orientation are considered, their geometry shows in Fig. 2(b). As detailed in Fig. 2(c), three H-shaped apertures are considered, the so-called external (placed at the right- and lefthand side) are identical while the dimensions of the internal one (placed in the middle of the wall) may be different.

As illustrated in Fig.2(a), all the four waveguide walls are perforated with 2 folded and $3 \mathrm{H}$-shaped slots, though their specific dimensions may be different. According to their orientation, the walls can be classified into two groups: the lateral walls (oriented along the YZ-plane), and a second group integrating the top and bottom walls (parallel to the XZ-plane). Under vertical excitation, the scattering properties of the cell are mainly determined by the lateral walls, since the impinging electric field is parallel to them. No interaction occurs with the top and bottom walls, since they are perpendicular to such excitation. In this case, the field direction imposes perfect electric conductor (PEC) boundaries on the top/bottom walls of the unit-cell, and perfect magnetic conductor (PMC) on the lateral ones. As a result, the resonators perforated on the top/bottom walls are short-circuited, and they have no influence in the wave scattering. Analogously, horizontal 


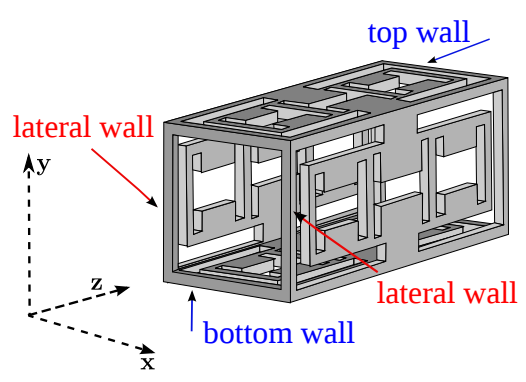

(a)

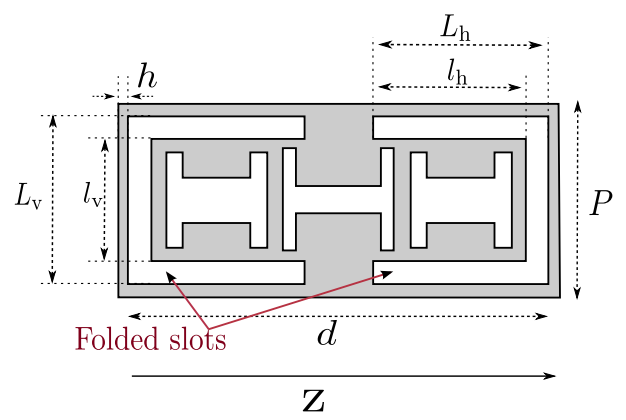

(b)

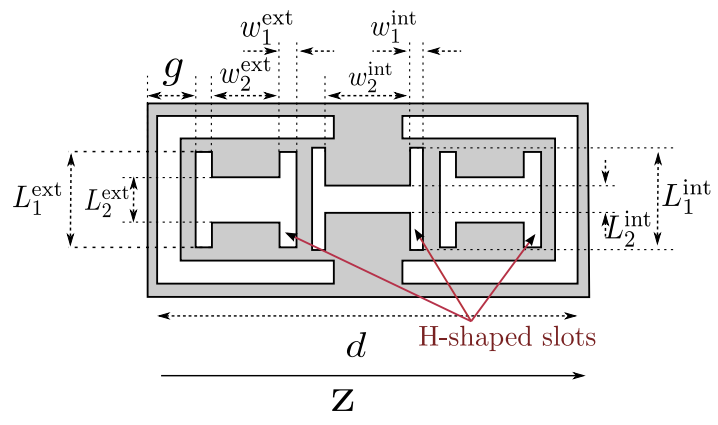

$(c)$
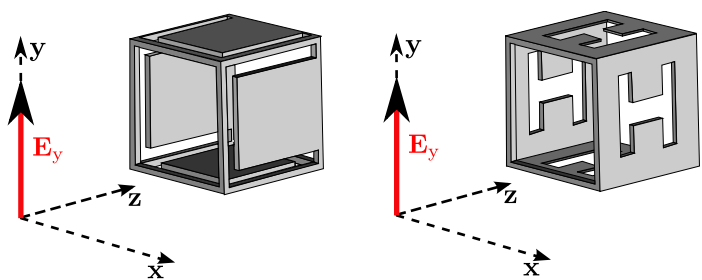

$(d)$

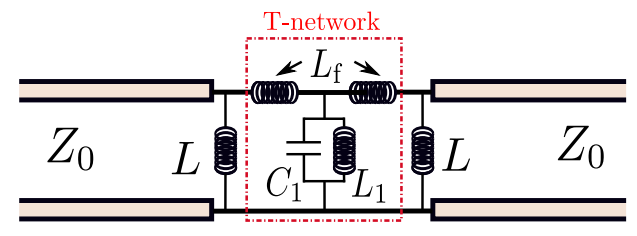

$(e)$

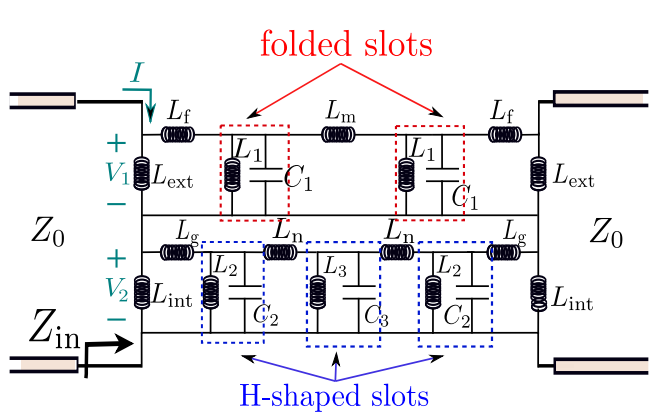

$(f)$

Fig. 2. Detail of the 3-D unit-cell and modelling. (a) View of the complete cell. (b) and (c) are identical and allow to define all the geometrical parameters of a wall. (d) Elementary cell containing a single resonator (either folded or H-shaped type). (e)Equivalent circuit for elementary cell in Fig. 2(d). (f) Equivalent circuit for the complete cell depicted in Fig. 2(a).

excitation interacts strongly with the top and bottom walls, since they are parallel to the electric-field vector. In such scenario, the lateral walls have no influence due to their perpendicular orientation.Therefore, the proposed geometry allows for independent interaction with vertical and horizontal excitation. In the following, an equivalent circuit model is proposed that allows to explain the unit-cell performance in detail.

The independent interaction with vertical and horizontal impinging fields allows to decompose the scenario of an arbitrary illumination into two orthogonal subproblems. Each of these is characterized by a different equivalent circuit: a first one for vertical incidence, where the corresponding circuit topology represents the behaviour of the lateral walls; and a second one for horizontal incidence considering the top and bottom walls. The circuit topology that we propose to model both scenarios is the same, and sketched in Fig. 2(e). However, the numerical values for the circuit elements may differ in both scenarios if the dimensions of the perforations in the walls are also different. In the following it is explained how this model is built.

It should be noted that, the previous virtual PEC/PMC walls would become periodic boundary conditions in the case of oblique incidence. Orthogonality between horizontal and vertical impinging electric fields would no longer be preserved. As a consequence, coupling would appear between the equivalent circuits characterizing both excitations, and between perforations occupying orthogonal walls. The model that is explained next will no longer be valid, since it assumes normal incidence. Extension of the circuit to account for oblique illumination, if possible, would significantly increase its complexity, and it is out of the scope of this manuscript.

\section{B. Circuit model topology}

When characterizing 2-D planar FSSs, it is common to adopt the waveguide-discontinuity approach, since there is no variation along the propagation direction (the $z$ axis) [38]. Such a discontinuity is then modelled by an equivalent

\section{ACCEPTED MANUSCRIPT}


lumped impedance accounting for the excitation of the infinite modal solutions supported on both sides of the discontinuity. Application of such an approach is not suited to the present case, since the 3-D unit-cell under consideration holds discrete perforations along $z$, thus not being uniform along the propagation direction. Proposed recently by the authors of this paper [3], we adopt the use of T-networks to model waveguide-atcutoff cells perforated in the $z$ direction. For the sake of clarity, this strategy is next explained by considering the elementary scenarios of Fig. 2(d), which contain a single perforation per wall (either folded- or H-shaped).

The circuit in Fig. 2(e) is proposed to characterize verticallypolarized excitation of the elementary cells in Fig. 2(d). Horizontal excitation would be modelled similarly. This T-network can be considered as an extension of the work done in [3]. The cell is built from a section of waveguide, whose periodicity is much smaller than half a wavelength. Therefore, the fundamental $\mathrm{TE}_{10}$ mode is below cutoff and its associated impedance is inductive [38]. The parallel connection between $C_{1}$ and $L_{1}$ in Fig. 2(e) represents the resonant character of the lateral perforations. The inductances $L_{\mathrm{f}}$ model the energy coupling from the impinging wave to the perforations. Such a coupling is highly reactive, and inductive, because it is done via the evanescent $\mathrm{TE}_{10}$ mode of the waveguide. Finally, the inductances $L$ placed at the input and output of the cell reproduce the effect of the aperture-discontinuity. An inductance is sufficient due to the subwavelength dimensions of the waveguide cross-section [38]. The red box highlighted in Fig. 2(e) can be considered as the contribution of a single resonator.

The complete cell of Fig.2(a) can be modelled by the circuit in Fig. 2(f), that is proposed as an intuitive extension of Fig.2(d). Such a circuit is the result of the cascading and series interconnection of several T-networks. To build the complete circuit model, the first main approximation consists of the connection in cascade of the T-models associated with resonators operating in the same frequency band. The second approximation is the series connection of the T-models associated to resonators operating in different frequency bands. Such approximations are based in the multi-resonant modelling done in [39]. More specifically, the set of two equal folded slots is represented by the upper block containing two cascaded identical T-networks (LC-tanks of lumped elements $L_{1}$ and $C_{1}$ ). Analogously, the group of three $\mathrm{H}$-shaped resonators is represented by the lower block containing three cascaded $\mathrm{T}$ networks (LC-tanks of lumped elements $L_{2}, C_{2}, L_{3}$ and $C_{3}$, since the first and last slots are considered to be identical). The inductive coupling coefficients are captured by the inductances $L_{\mathrm{f}}, L_{\mathrm{m}}, L_{\mathrm{g}}$ and $L_{\mathrm{n}}$. Finally, the inductances $L_{\mathrm{ext}}$ and $L_{\text {int }}$ represent the presence of the waveguide aperture. Possible mutual coupling between folded and $\mathrm{H}$-shaped slots is not expected to be significant within the structure bands of operation, and thus not included in the proposed circuit. This assumption is justified by the fact that such bands are mainly determined by the resonance character of a given type of perforation. In this case, and as it is explained in detail in [40] for the case of compound grating modelling, mutual coupling is almost negligible and its consideration increases drastically the complexity of the circuit. In Fig. 2(f), the cell input impedance $Z_{\text {in }}$ is defined, together with the voltages $V_{1 / 2}$ and the current $I$. These electrical parameters allow to define the following branch impedances

$$
Z_{i n, 1 / 2}=V_{1 / 2} / I \text {. }
$$

which capture, respectively, information from the upper/bottom branches of elements (folded/H-shaped slots) in the circuit of Fig. 2(f).

The numerical values of each element in the circuit of Fig. 2(d) are obtained in two steps. In the first one, we propose to base on the elementary circuits in Fig. 2(d) to obtain the T-network associated with each perforation. The set of parameters $\left[L_{1}, C_{1}\right.$ and $\left.L_{\mathrm{f}}\right],\left[L_{2}, C_{2}\right.$ and $\left.L_{\mathrm{g}}\right]$ and $\left[L_{3}, C_{3}\right.$ and $L_{\mathrm{n}}$ ] can be obtained independently by numerical fitting the results provided by the circuit model in Fig. 2(e) with a full-wave solver. Similarly as it was done in [3], only two frequency points need to be simulated to obtain each set of parameters. In the second step, the complete circuit of Fig. 2(f) is considered, and a final optimization is performed to achieve the proper values of the coupling inductances $L_{\mathrm{f}}, L_{\mathrm{m}}, L_{\mathrm{g}}$ and $L_{\mathrm{n}}$.

\section{Circuit model validation}

To check the validity of the circuit model, an example of unit-cell is analysed which dimensions are detailed in TABLE I. For simplicity, the four walls of this cell are chosen to be identical, thus identical behaviour is expected for both vertical and horizontal excitation. The corresponding scattering parameters are plotted in Fig. 3 with respect to the normalized wavelength $\lambda$. The axis normalization is done with respect to the upper band central wavelength $\lambda_{0}$, which is considered as a reference. The magnitude and phase of the transmission coefficient are shown respectively in Fig. 3(a) and Fig. 3(b). Both the results obtained by the equivalent circuit and the full-wave solver are shown for comparison. Two broad transmission passbands can be observed in Fig. 3(a), which are centred respectively around $1.5 \lambda_{0}$ and $\lambda_{0}$. These two passbands constitute the dual-band behaviour of the proposed unit-cell, and they are created between two peaks of total transmission. A third and narrow-band peak can be also observed at around $1.2 \lambda_{0}$; this one is is considered as spurious.

The appearance of the two passbands is directly related to the resonance of the LC-tanks in Fig. 2(d). As explained above, such LC-tanks characterize the first resonance of the slots in the cell, and they appear when their length is approximately half a wavelength. By considering the slots dimensions in TABLE I, it is straightforward to verify that the appearance of the lowest transmission band is associated with the resonances of the folded-slots (the longest ones in the cell) and that the highest band is associated with the resonances of the $\mathrm{H}$ shaped slots (whose length is shorter). It should be noted in Fig. 3(b) that the phase of the transmission coefficient presents an almost linear behaviour within the regions shadowed in green. This feature is exploited in the next section for the synthesis of polarization conversion.

Good agreement can be observed between the predictions given by circuit model and the full-wave solver results. The

\section{ACCEPTED MANUSCRIPT}




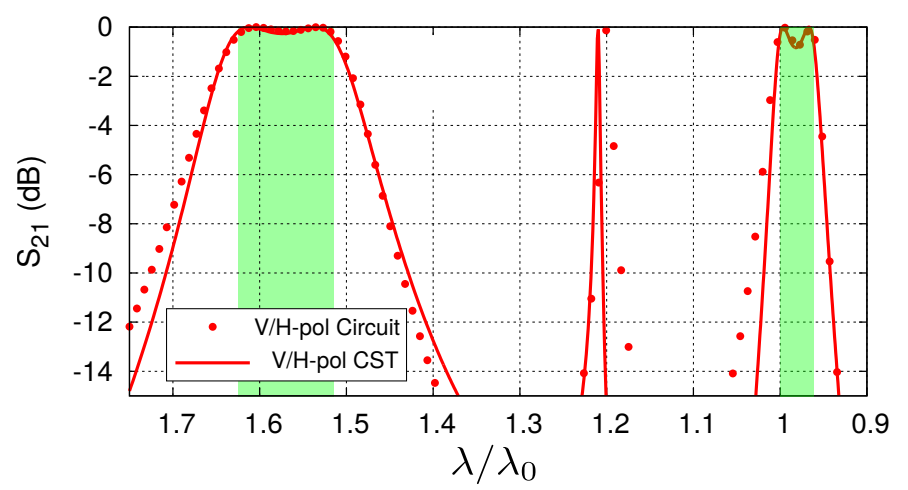

(a)

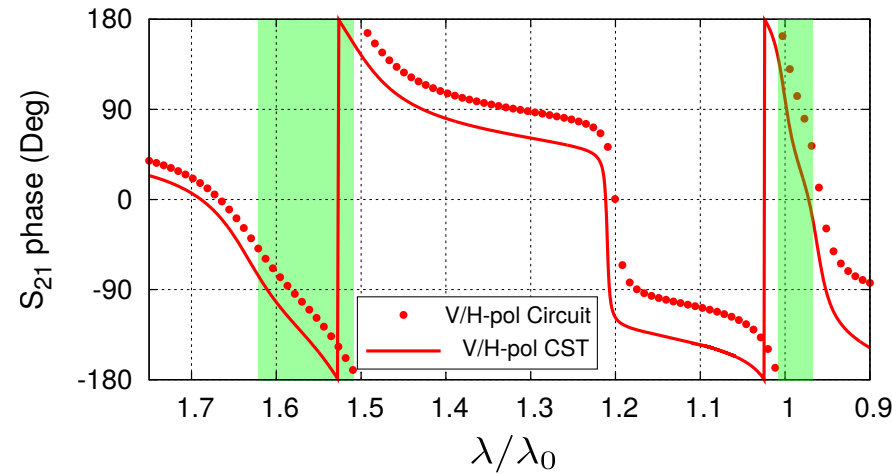

(b)

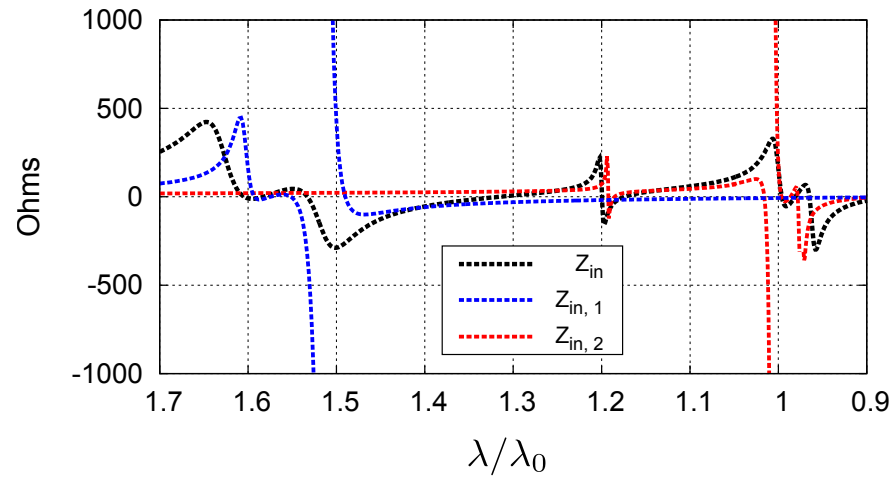

(c)

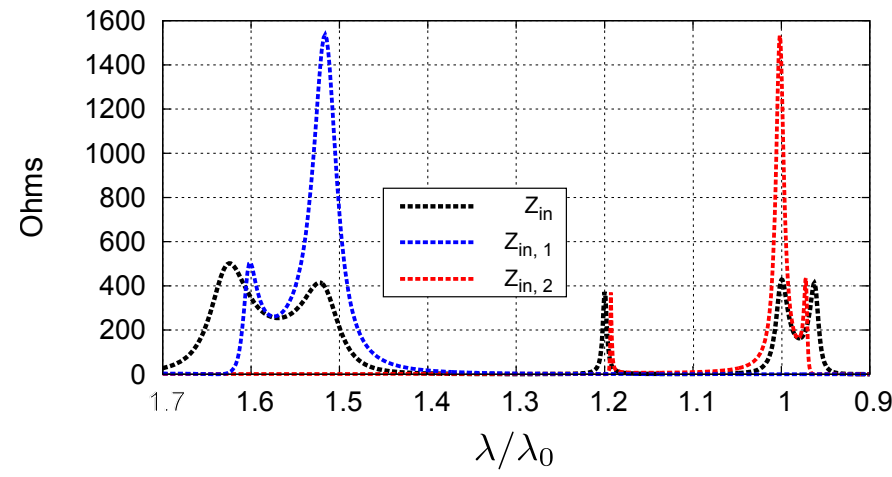

(d)

Fig. 3. Cell transmission coefficient (a) magnitude and (b) phase. (c) Imaginary and (d) real part of the input impedance and branches impedances defined in (1). Cell dimensions are $P=0.57 \lambda_{0}, t=0.08 \lambda_{0}, d=1.3 \lambda_{0}$. The dimensions of the slots are detailed in TABLE I.

dual-band behaviour is reproduced well by the model. Some minor inaccuracies appear around the second passband, mainly
TABLE I

PARAMETERS FOR THE DESIGN OF FIG. 3

\begin{tabular}{|c|c|c|c|c|}
\hline \multicolumn{5}{|c|}{ Vertical polarization } \\
\hline $\begin{array}{l}\text { Lateral } \\
\text { resonators }\end{array}$ & $\begin{array}{l}\text { Normalized } \\
\lambda_{0} \\
\end{array}$ & $\begin{array}{l}\text { Value }(\mathrm{mm}) \\
(15.2 \mathrm{GHz})\end{array}$ & $\begin{array}{l}\text { Circuit } \\
\text { element }\end{array}$ & \begin{tabular}{|l|} 
Value \\
$(15.2 \mathrm{GHz})$ \\
\end{tabular} \\
\hline$L_{\mathrm{v}}$ & 0.50 & 9.80 & $L_{\mathrm{ext}}$ & $2.20 \mathrm{nH}$ \\
\hline$l_{\mathrm{v}}$ & 0.37 & 7.20 & $L_{\text {int }}$ & $2.40 \mathrm{nH}$ \\
\hline$L_{\mathrm{h}}$ & 0.53 & 10.40 & $L_{\mathrm{f}}$ & $0.15 \mathrm{nH}$ \\
\hline$l_{\mathrm{v}}$ & 0.46 & 9.00 & $L_{\mathrm{g}}$ & $0.59 \mathrm{nH}$ \\
\hline$h$ & 0.03 & 0.60 & $L_{\mathrm{m}}$ & $5.90 \mathrm{nH}$ \\
\hline$L_{1}^{\mathrm{ext}}$ & 0.28 & 5.60 & $L_{\mathrm{n}}$ & $1.57 \mathrm{nH}$ \\
\hline$L_{2}^{\frac{1}{\mathrm{ext}}}$ & 0.14 & 2.70 & $C_{1}$ & $581 \mathrm{fF}$ \\
\hline$L_{1}^{\text {int }}$ & 0.30 & 6.00 & $L_{1}$ & $0.65 \mathrm{nH}$ \\
\hline$L_{2}^{\text {int }}$ & 0.08 & 1.60 & $C_{2}$ & $553 \mathrm{fF}$ \\
\hline$w_{1}^{\text {ext }}$ & 0.05 & 1.00 & $L_{2}$ & $0.25 \mathrm{nH}$ \\
\hline$w_{2}^{\text {ext }}$ & 0.20 & 4.00 & $C_{3}$ & $668 \mathrm{fF}$ \\
\hline$w_{1}^{\text {int }}$ & 0.04 & 0.80 & $L_{3}$ & $0.29 \mathrm{nH}$ \\
\hline$w_{2}^{\text {int }}$ & 0.25 & 5.00 & $Z_{0}$ & $377 \Omega$ \\
\hline$g$ & 0.14 & 2.80 & & \\
\hline \multicolumn{5}{|c|}{ Horizontal polarization } \\
\hline $\begin{array}{l}\text { Top/bottom } \\
\text { resonators }\end{array}$ & $\begin{array}{l}\text { Normalized } \\
\lambda_{0}\end{array}$ & $\begin{array}{l}\text { Value }(\mathrm{mm}) \\
(15.2 \mathrm{GHz})\end{array}$ & $\begin{array}{l}\text { Circuit } \\
\text { element }\end{array}$ & \begin{tabular}{|l|} 
Value \\
$(15.2 \mathrm{GHz})$
\end{tabular} \\
\hline$L_{\mathrm{V}}$ & 0.50 & 9.80 & $L_{\text {ext }}$ & $2.20 \mathrm{nH}$ \\
\hline$l_{\mathrm{v}}$ & 0.37 & 7.20 & $L_{\text {int }}$ & $2.40 \mathrm{nH}$ \\
\hline$L_{\mathrm{h}}$ & 0.53 & 10.40 & $L_{\mathrm{f}}$ & $0.15 \mathrm{nH}$ \\
\hline$l_{\mathrm{v}}$ & 0.46 & 9.00 & $L_{\mathrm{g}}$ & $0.59 \mathrm{nH}$ \\
\hline$h$ & 0.03 & 0.06 & $L_{\mathrm{m}}^{\circ}$ & $5.90 \mathrm{nH}$ \\
\hline$L_{1}^{\mathrm{ext}}$ & 0.28 & 5.60 & $L_{\mathrm{n}}$ & $1.57 \mathrm{nH}$ \\
\hline$L_{2}^{\mathrm{ext}}$ & 0.14 & 2.70 & $C_{1}$ & $581 \mathrm{fF}$ \\
\hline$L_{1}^{\text {int }}$ & 0.30 & 6.00 & $L_{1}$ & $0.65 \mathrm{nH}$ \\
\hline$L_{2}^{\text {int }}$ & 0.08 & 1.60 & $C_{2}$ & $553 \mathrm{fF}$ \\
\hline$w_{1}^{\text {ext }}$ & 0.05 & 1.00 & $L_{2}$ & $0.25 \mathrm{nH}$ \\
\hline$w_{2}^{\mathrm{ext}}$ & 0.20 & 4.00 & $C_{3}$ & $668 \mathrm{fF}$ \\
\hline$w_{1}^{\text {int }}$ & 0.04 & 0.80 & $L_{3}$ & $0.29 \mathrm{nH}$ \\
\hline$w_{2}^{\text {int }}$ & 0.25 & 5.00 & $Z_{0}$ & $377 \Omega$ \\
\hline$g$ & 0.14 & 2.80 & & \\
\hline
\end{tabular}

manifested in the phase-curve. This fact remarks the limit of validity of the model. As the normalized wavelength decreases (increase of frequency), dynamical effects begin to appear which are mainly related to the the excitation of higher-order resonances in the slots. The relevance of the proposed circuit topology is also validated by the prediction of the spurious transmission peak, which is next explained.

The values obtained for the circuit input impedance, and those defined in (1) are plotted in Fig.3(c) and Fig.3(d). Inspection of such figures allows to check that the evolution of $Z_{\text {in }}$ is closely linked to the individual evolution of $Z_{\text {in }, 1}$ and $Z_{\text {in, } 2}$. For the lower band, the imaginary part of $Z_{\text {in }}$ becomes null whereas the real part is very close to the value of the input transmission line $Z_{0}=376.73 \Omega$. Good matching is therefore achieved in this band, and high levels of transmission. The shape of the curve within this frequency region is quite similar to that of $Z_{\text {in, } 1}$, mainly associated with the set of folded slots. It can be therefore stated that, in the vicinity of their resonance, the folded slots govern the unit-cell scattering parameters. The same rationale is applied for the higher band, where the evolution of $Z_{\text {in }}$ resembles that of $Z_{\mathrm{in}, 2}$, which is mainly associated with the set of $\mathrm{H}$-shaped resonators. Finally, the narrow-band peak at $1.2 \lambda_{0}$ is apparently coming from a singularity of $Z_{\text {in,2 }}$. Therefore, it can be deduced from the circuit that the spurious resonance may be caused by a resonance of the $\mathrm{H}$-shaped slots influenced by the presence of the folded ones. 


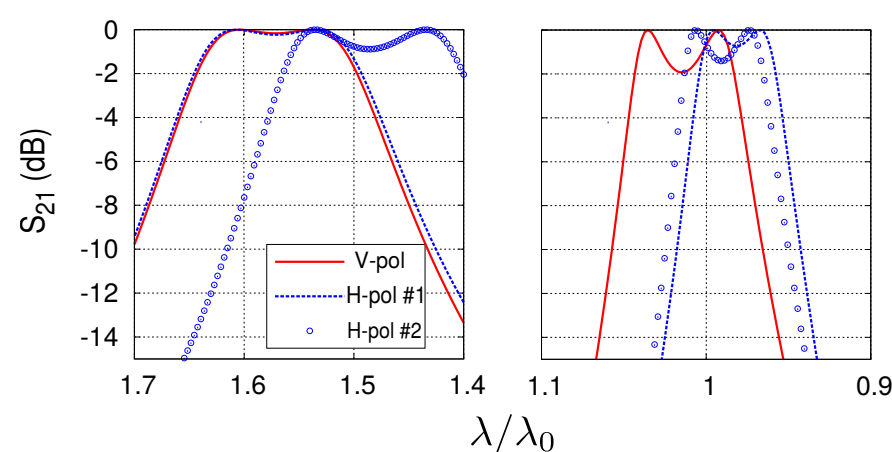

(a)

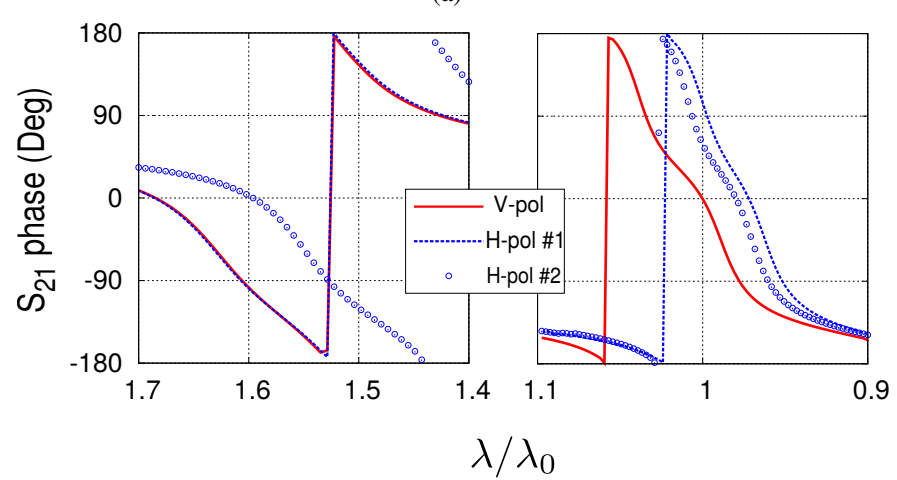

(b)

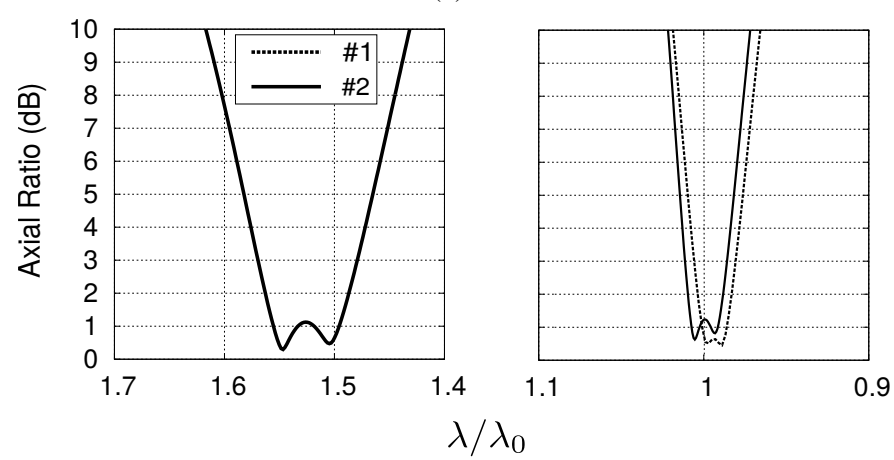

(c)

Fig. 4. Transmission coefficient (a) magnitude and (b) phase. (c) Axial ratio. Cell dimensions: $P=0.57 \lambda_{0}, t=0.08 \lambda_{0}, d=1.3 \lambda_{0}$.

Finally, it should be noted that the physical principle underlying the operation of this 3-D structure could be exploited to match operation frequencies and requirements different than those considered in this manuscript. Not only can the dimensions be scaled (this is why normalization with respect to $\lambda_{0}$ is considered), but also loading the unit cell with a greater number of resonating elements, or with different geometries, can be considered to suit specific manufacturing approaches. This way, we could expect feasible operation at millimetre waves in a near future, by adapting the design, and employing new 3D-micro/nano printers or other sophisticated micro-machining techniques.

\section{Versatility OF THE UNIT CELL}

This section illustrates how the proposed unit-cell allows one to arbitrarily set the polarization of the wave radiated through the FSS at both passbands. The example considered

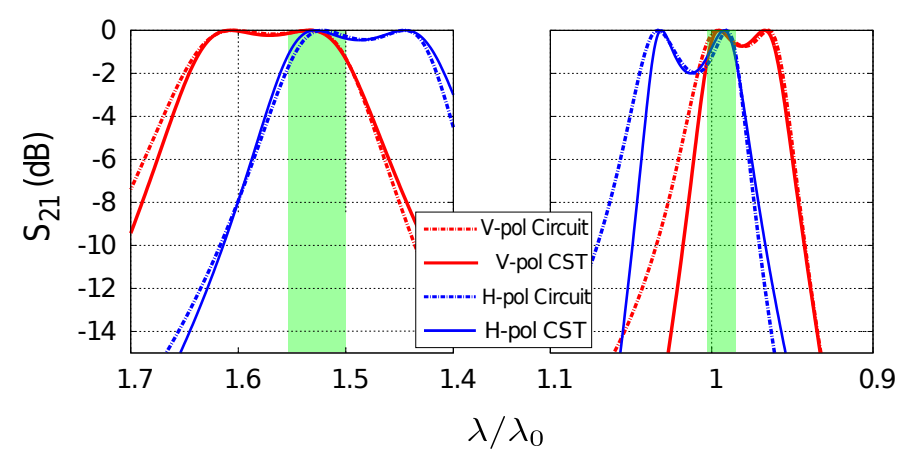

(a)

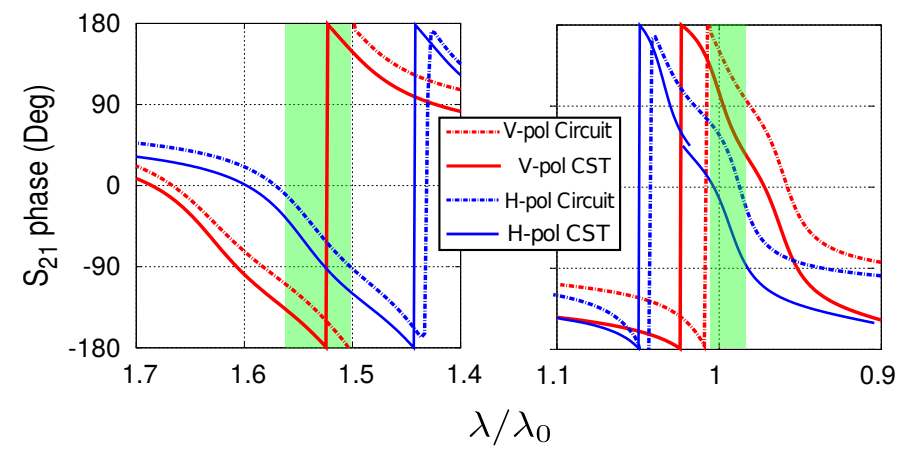

(b)

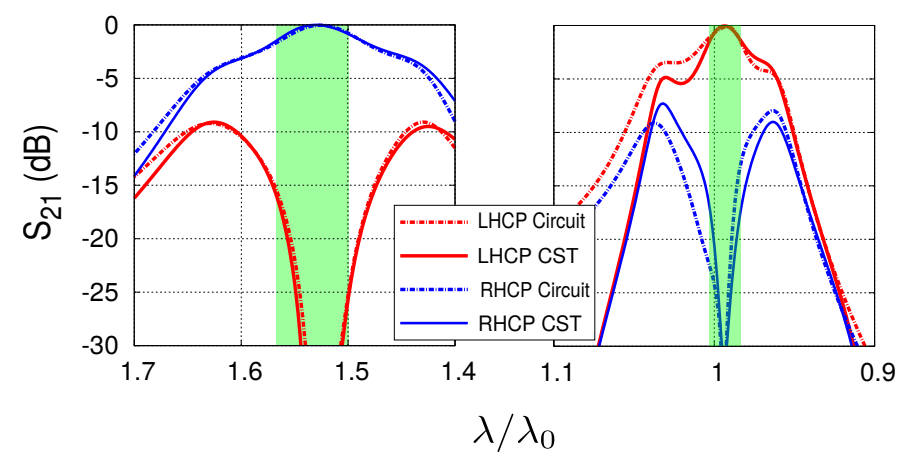

(c)

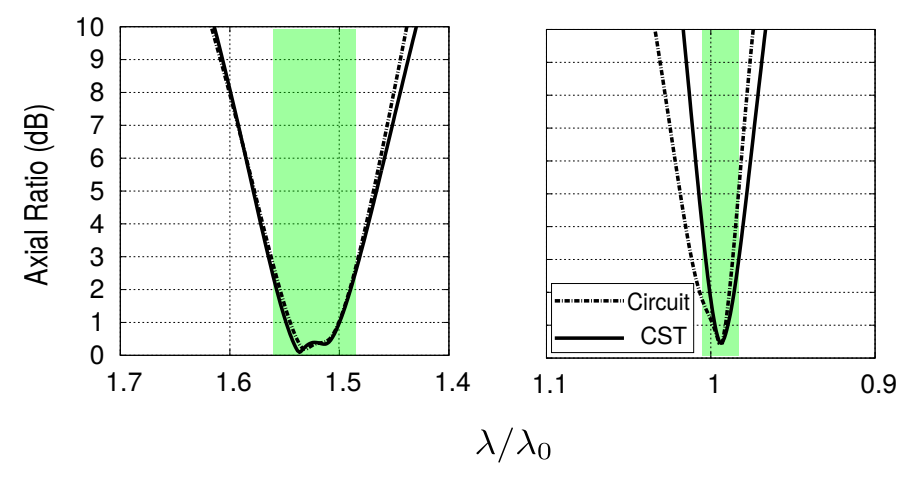

(d)

Fig. 5. Transmission coefficient (a) magnitude and (b) phase. (c) Axial ratio. Cell dimensions: $P=0.57 \lambda_{0}, t=0.08 \lambda_{0}, d=1.3 \lambda_{0}$. The dimensions of the slots are detailed in TABLE II.

in the previous section is the simplest one, which consists of a unit-cell whose lateral walls are equal to the top/bottom ones. Since the cell behaves identically for vertical and horizontal polarization, the polarization is not altered in any of the bands. This example represents a dual-polarized frequency selective 
filter with a relative distance between the bands defined by a ratio of about 1.5 .

The possibility to interact independently with vertical and horizontal polarization at each band can be exploited for different applications related to polarization conversion. In Fig. 4, two illustrative examples are considered. The first example, denoted as \#1, consists of a dual-band device transmitting dual-linear polarization in the lowest band and inducing $\mathrm{CP}$ in the highest one. The second example, denoted as \#2, consists of a dual-band device inducing right-hand $(\mathrm{RH}) \mathrm{CP}$ in both passbands. A third example, denoted as \#3, is considered in Fig. 5. This last design induces orthogonal sense of $\mathrm{CP}$ at each band. In all examples, the illumination is considered to be slanted of $45^{\circ}$ with respect the $x$-cartesian axis. This is the common excitation of polarizers [3], since it provides simultaneous excitation of in-phase vertical and horizontal field components with equal magnitude.

TABLE II

PARAMETERS FOR THE DESIGN OF FIG. 5

\begin{tabular}{|l|l|l||l|l|}
\hline \multicolumn{5}{|c|}{ Vertical polarization } \\
\hline Lateral & Normalized & Value $(\mathrm{mm})$ & Circuit & Value \\
resonators & $\lambda_{0}$ & $(15.2 \mathrm{GHz})$ & element & $(15.2 \mathrm{GHz})$ \\
\hline$L_{\mathrm{v}}$ & 0.50 & 9.80 & $L_{\text {ext }}$ & $2.20 \mathrm{nH}$ \\
$l_{\mathrm{v}}$ & 0.37 & 7.20 & $L_{\text {int }}$ & $2.40 \mathrm{nH}$ \\
$L_{\mathrm{h}}$ & 0.53 & 10.40 & $L_{\mathrm{f}}$ & $0.15 \mathrm{nH}$ \\
$l_{\mathrm{v}}$ & 0.46 & 9.00 & $L_{\mathrm{g}}$ & $0.59 \mathrm{nH}$ \\
$h$ & 0.03 & 0.60 & $L_{\mathrm{m}}$ & $5.90 \mathrm{nH}$ \\
$L_{1}^{\text {ext }}$ & 0.28 & 5.60 & $L_{\mathrm{n}}$ & $1.57 \mathrm{nH}$ \\
$L_{2}^{\text {ext }}$ & 0.14 & 2.70 & $C_{1}$ & $581 \mathrm{fF}$ \\
$L_{1}^{\text {int }}$ & 0.30 & 6.00 & $L_{1}$ & $0.65 \mathrm{nH}$ \\
$L_{2}^{\text {int }}$ & 0.08 & 1.60 & $C_{2}$ & $552 \mathrm{fF}$ \\
$w_{1}^{\text {ext }}$ & 0.05 & 1.00 & $L_{2}$ & $0.25 \mathrm{nH}$ \\
$w_{2}^{\text {ext }}$ & 0.20 & 4.00 & $C_{3}$ & $668 \mathrm{fF}$ \\
$w_{1}^{\text {int }}$ & 0.04 & 0.80 & $L_{3}$ & $0.29 \mathrm{nH}$ \\
$w_{2}^{\text {int }}$ & 0.25 & 5.00 & $Z_{0}$ & $377 \Omega$ \\
$g$ & 0.14 & 2.80 & & \\
\hline \multicolumn{5}{|c|}{ Horizontal polarization } \\
\hline Top/bottom & Normalized & Value $(\mathrm{mm})$ & Circuit & Value \\
resonators & $\lambda_{0}$ & $(15.2 \mathrm{GHz})$ & element & $(15.2 \mathrm{GHz})$ \\
\hline$L_{\mathrm{v}}$ & 0.50 & 9.85 & $L_{\text {ext }}$ & $2.20 \mathrm{nH}$ \\
$l_{\mathrm{v}}$ & 0.36 & 7.00 & $L_{\text {int }}$ & $2.40 \mathrm{nH}$ \\
$L_{\mathrm{h}}$ & 0.50 & 9.85 & $L_{\mathrm{f}}$ & $0.34 \mathrm{nH}$ \\
$l_{\mathrm{v}}$ & 0.45 & 8.93 & $L_{\mathrm{g}}$ & $0.73 \mathrm{nH}$ \\
$h$ & 0.04 & 0.08 & $L_{\mathrm{m}}$ & $5.90 \mathrm{nH}$ \\
$L_{1}^{\text {ext }}$ & 0.29 & 5.80 & $L_{\mathrm{n}}$ & $1.60 \mathrm{nH}$ \\
$L_{2}^{\text {ext }}$ & 0.12 & 2.40 & $C_{1}$ & $552 \mathrm{fF}$ \\
$L_{1}^{\text {int }}$ & 0.30 & 6.00 & $L_{1}$ & $0.59 \mathrm{nH}$ \\
$L_{2}^{\text {int }}$ & 0.08 & 1.60 & $C_{2}$ & $573 \mathrm{fF}$ \\
$w_{1}^{\text {ext }}$ & 0.05 & 1.00 & $L_{2}$ & $0.26 \mathrm{nH}$ \\
$w_{2}^{\text {ext }}$ & 0.20 & 4.00 & $C_{3}$ & $686 \mathrm{fF}$ \\
$w_{1}^{\text {int }}$ & 0.04 & 0.8 & $L_{3}$ & $0.28 \mathrm{nH}$ \\
$w_{2}^{\text {int }}$ & 0.24 & 4.80 & $Z_{0}$ & $377 \Omega$ \\
$g$ & 0.14 & 2.70 & & \\
\hline
\end{tabular}

In design \#1, folded slots on lateral and top/bottom walls remain identical. Thus, as it can be expected, the lower passbands in Fig. 4(a) (where $\lambda \approx 1.5 \lambda_{0}$ ) do overlap completely for horizontal and vertical polarization, since they are associated with the resonances of folded slots. No polarization conversion is here attained since the phase responses do also overlap. However, some relative differences exist in the dimensions of the $\mathrm{H}$-shaped slots. More specifically, H-shaped slots on top/bottom walls are smaller than the ones on the lateral walls. Therefore, in the upper band of Fig. 4(a) (where

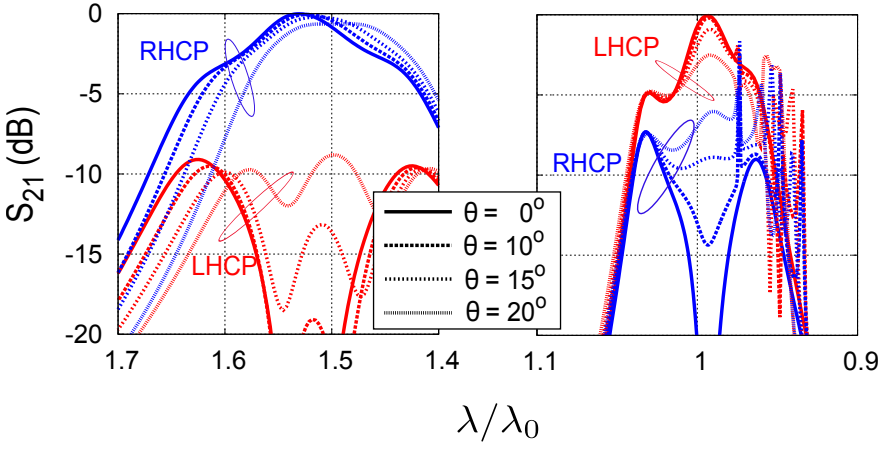

(a)

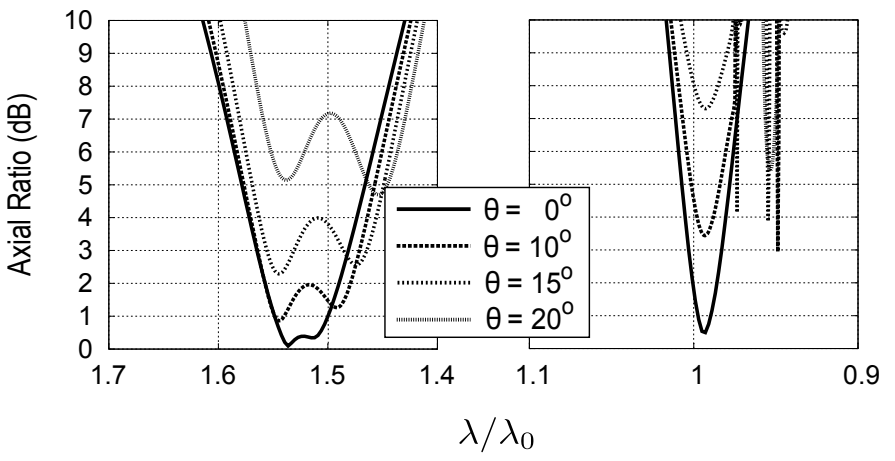

(b)

Fig. 6. Performance under oblique plane-wave incidence of the design considered in Fig. 5. (a) Magnitude of transmission coefficient (b) Axial ratio.

$\lambda \approx \lambda_{0}$ ), the passband for horizontal polarization is slightly shifted towards the right and there is only partial overlapping between both passbands where $S_{11}>-1 \mathrm{~dB}$. Within this region, a relative phase difference of $90^{\circ}$ can be observed between horizontal and vertical polarization in Fig.4(b). This phase difference remains stable in the shadowed region due to the linearity of the phase inside the passbands. CP is consequently induced in such band. The corresponding axial ratio (AR) shows in Fig. 4(c), which denotes good purity for the higher band. No AR results are shown for this example in the lower band, since the design preserves the dual-linear illumination, the corresponding numerical results are close to infinite values.

In design \#2, both the folded slots and the H-shaped slots are shorter on the top/bottom walls than those on the lateral walls. Therefore, both in the upper and lower bands of Fig. 4(a), the passband for horizontal polarization is slightly shifted towards the right and there is partial overlapping with respect to the transmission of vertical polarization. Within this region, a relative phase difference of $90^{\circ}$ can be observed between horizontal and vertical polarization in both bands of Fig. 4(b). RHCP is consequently induced in both bands, as it is proven by the good AR results obtained for both bands in Fig. 4(c).

Finally, in design \#3, H-shaped slots in the lateral walls are again shorter than those on top/bottom walls. However, the opposite feature characterizes folded slots, which are longer in the lateral walls than those on top/bottom walls. This geometry has associated the relative shift between vertical and horizontal passbands that can be observed in Fig. 5(a). The horizontal passband is shifted to the left in the lower band with respect to the vertical polarization, and to the right in the higher band. 
TABLE III

PERFormance BENCHMARKING OF POLARIZING STRUCTURES. IL STANDS FOR INSERTION LOSSES, BW FOR FRACTIONAL BANDWIDTH, $\lambda$ FOR THE WAVELENGTH IN THE LOWER FREQUENCY OF OPERATION, $P$ FOR THE PERIODICITY OF THE UNIT-CELL, T FOR TRANSMISSION AND R FOR REFLECTION.

\begin{tabular}{|l|l|l|l|l|l|l|l|l|l|}
\hline Ref. & $\begin{array}{l}\text { Center Freq. } \\
(\mathrm{GHz})\end{array}$ & $\begin{array}{l}\text { IL } \leq 1 \mathrm{~dB} \\
\mathrm{BW}(\%)\end{array}$ & $\begin{array}{l}\text { AR } \leq 3 \mathrm{~dB} \\
\mathrm{BW}(\%)\end{array}$ & $\begin{array}{l}\text { Thickness } \\
(\lambda)\end{array}$ & $P(\lambda)$ & $\begin{array}{l}\text { Number of } \\
\text { layers }\end{array}$ & $\begin{array}{l}\text { Reverse } \\
\text { sense of CP }\end{array}$ & $\begin{array}{l}\text { Use of } \\
\text { dielectric }\end{array}$ & $\begin{array}{l}\text { Operation } \\
\text { mode }(\mathrm{T} / \mathrm{R})\end{array}$ \\
\hline$[19]$ & $\begin{array}{l}15 \\
\text { (single band) }\end{array}$ & 57 & 66 & 0.50 & 0.26 & 5 & No & Yes & $\mathrm{T}$ \\
\hline$[20]$ & $12 / 18.5$ & - & $16.6 / 27$ & 0.06 & 0.32 & 1 & Yes & Yes & $\mathrm{R}$ \\
\hline$[21]$ & $17.5 / 28$ & - & $40 / 14$ & 0.09 & 0.57 & 1 & Yes & Yes & $\mathrm{R}$ \\
\hline$[22]$ & $\begin{array}{l}8.75 \\
\text { (single band) }\end{array}$ & 17 & 17 & 0.04 & 0.40 & 2 & No & Yes & $\mathrm{T}$ \\
\hline$[28]$ & $19.95 / 29.75$ & $11.6 / 6.6$ & $2.5 / 1.7$ & 0.07 & 0.35 & 3 & Yes & Yes & $\mathrm{T}$ \\
\hline$[30]$ & $18.5 / 29$ & - & $29 / 12$ & 0.10 & 0.25 & 2 & Yes & Yes & $\mathrm{T}$ \\
\hline$[29]$ & $19.6 / 29.6$ & $14.6 / 6.8$ & $4 / 2.7$ & 0.31 & - & 5 & No & Yes & $\mathrm{T}$ \\
\hline$[31]$ & $18.5 / 27.75$ & $4.5 / 5.5$ & $4.5 / 5.5$ & 0.10 & 0.49 & 3 & Yes & Yes & $\mathrm{T}$ \\
\hline$[41]$ & $11.6 / 13.2$ & - & - & 0.03 & 0.19 & 2 & Yes & Yes & $\mathrm{T}$ \\
\hline This work & $10 / 15.2$ & $3.5 / 3.2$ & $5.8 / 2.6$ & 1.30 & 0.57 & 1 & Yes & No & $\mathrm{T}$ \\
\hline
\end{tabular}

The partial-overlapping regions have been shadowed in green in Fig. 5. Within these regions, the associated phase curves in Fig. 5(b) show relative phase delays of $90^{\circ}$ with opposite sign at each band. The associated transmission coefficient expressed in terms of the states of circular polarization (either RHCP or LHCP [25]), has been computed and shown in Fig. 5(c). In the lower band, RHCP is induced with a negligible cross-polarization component. LHCP is induced in the higher band, where RHCP is negligible. The corresponding AR is plotted in Fig. 5(d), which shows good levels in both bands. In this last example, the results given by the circuit model have also been plotted, proving very good agreement with the fullwave solver. TABLE II shows the specific values associated with the circuit elements and geometry of the unit-cell.

In TABLE III the performance of design \#3 is compared to other polarizing devices available in the literature, providing single or dual-band behaviour. This table shows that singleband designs are the ones allowing for polarization conversion in a wider bandwidth. The operation bandwidth of the present prototype is comparable to the rest of examples operating in dual-band, and no remarkable advantage is appreciated in terms of RF performance. Though its thickness is larger than the rest, no dielectric materials are employed in our case. From this table it can be concluded that no other proposal allows yet for $\mathrm{CP}$ conversion avoiding the need for dielectric materials. This feature constitutes the main advantage of this solution.

For the sake of completeness, the behaviour of design \#3 is computed for oblique plane-wave incidence and shown in Fig. 6. This figure shows that the magnitude of the transmission coefficient is significantly impacted by the inclination of the impinging field, making the unit-cell less transparent and deteriorating the cross-polarization levels. Such degradation is also reflected in the AR subfigure. These results confirm that the present 3-D FSS is conceived to operate under normal incidence. In the need for beam scanning capabilities, this architecture could be useful in applications where the system is scanned mechanically, such as in the examples exposed in [35] or [42].

\section{EXPERIMENTS}

In order to validate the concepts reported in the paper, a dual-band polarizer with opposite polarization conversion in each band (the example considered in Fig. 5) has been manufactured and tested. The lowest frequency has been fixed around $10 \mathrm{GHz}$, with the highest frequency around $15 \mathrm{GHz}$ (the centre of the band is in $15.2 \mathrm{GHz}$, where $\lambda_{0}$ is defined).

An arrangement of $7 \times 7$ unit-cells has been fabricated in aluminium using wire electrical discharge machining (EDM). This technique allows to obtain a monolithic object as well as offers very good manufacturing tolerances $( \pm 1 \mathrm{um})$. Other manufacturing approaches have been also considered and discarded, either for involving complicated assemblies (CNC milling) or not being suited for the type of geometry under consideration (as stereolithography or selective laser melting 3D-printing). The manufactured polarizer is shown in Fig. 7 (left and center subfigures). The overall dimensions are 81.4 $\mathrm{mm} \times 81.4 \mathrm{~mm} \times 26 \mathrm{~mm}\left(2.7 \lambda_{0} \times 2.7 \lambda_{0} \times 0.87 \lambda_{0}\right)$, while the detailed dimensions can be found in Table II.

The experiments have been carried out using a near-field multi-probe scanner for antenna pattern measurements (MVG StarLab chamber [43], whose operation range is 0.6 to 18 $\mathrm{GHz}$ ). The details of the device under test (DUT) can be seen in Fig. 7 (right subfigure) while its positioning within the chamber is shown in Fig. 8. The DUT consists of a linearlypolarized horn that illuminates the polarizer, which is rotated $45^{\circ}$ with respect to the input of the horn. The polarizer is held at a given distance from the aperture of the horn by a custom support (its arms are convex to minimize diffraction and possible interaction with the horn radiation). Both the horn and the support have been 3D-printed using stereolithography (SLA) [44]. While the horn has been metallized to be RF-conductive (standard process for SLA components), the support and the screws used to hold the polarizer have not been metallized to minimize diffraction and their influence in the measurement. In order to add some redundancy to the experiments, two spacers of have been manufactured that assure a spacing between the polarizer and the horn aperture of $10 \mathrm{~mm}$ and $30 \mathrm{~mm}$, respectively. The overall size of the DUT is $17 \mathrm{~cm} \mathrm{x} 17 \mathrm{~cm}$ $x 19.5 \mathrm{~cm}$ (considering the use of the bigger spacer). All the items have been manufactured by the company SWISSto12.

The horn is designed basing on classical guidelines [45]; its aperture diameter measures $50 \mathrm{~mm}$, which corresponds to a broadside gain of $14 \mathrm{~dB}$ at $10 \mathrm{GHz}$ and $17.3 \mathrm{~dB}$ at 15.2 GHz. Such design assures that the power level reaching the

\section{ACCEPTED MANUSCRIPT}



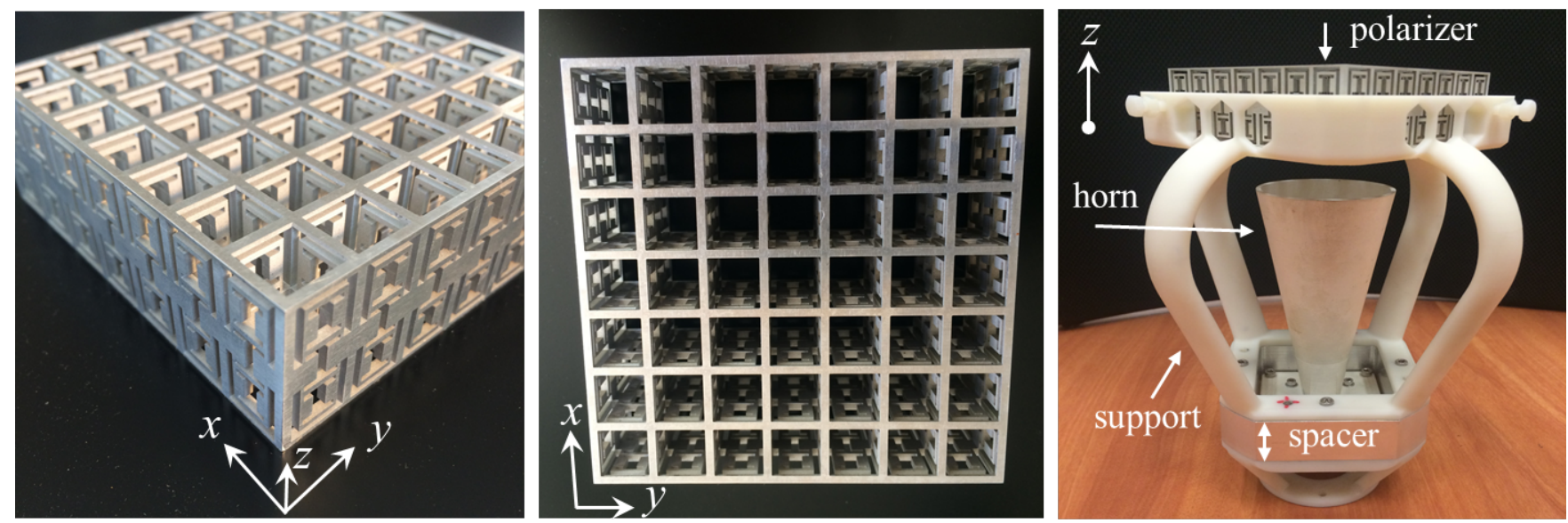

Fig. 7. Photographs of the monolithic polarizer. On the left is the 3D view of the prototype, on the center the view from the top, and on the right is the conceived DUT conceived to measure the polarizer illuminated by a horn.

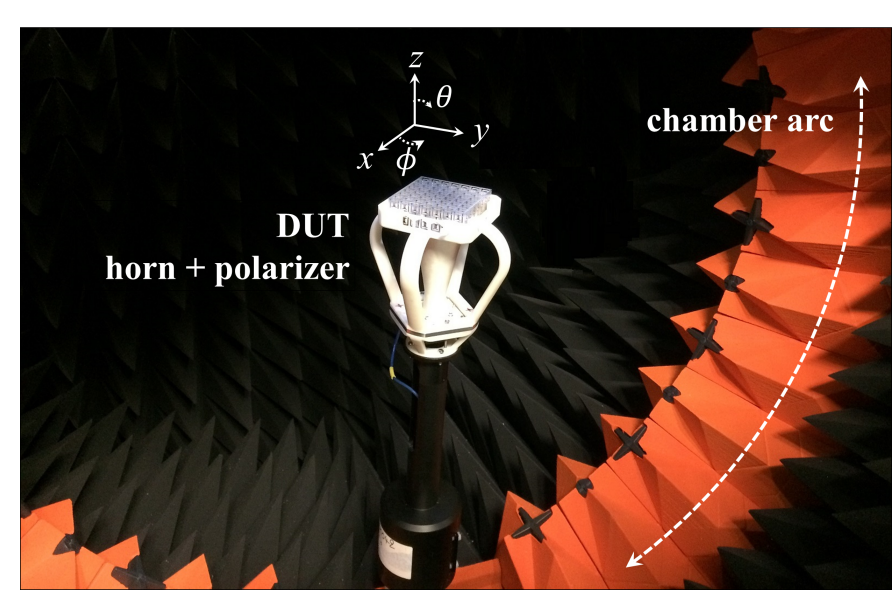

Fig. 8. DUT in the near-field multi-probe chamber.

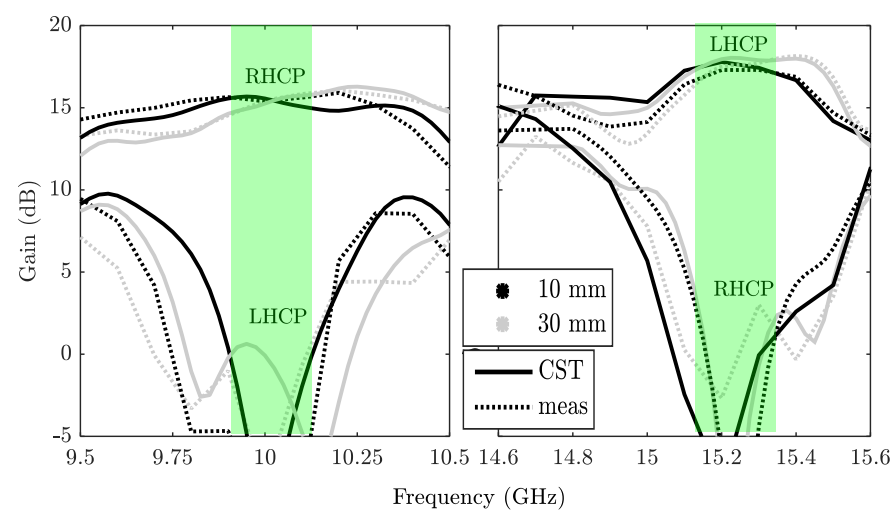

Fig. 9. Gain at broadside of the polarizer illuminated by the horn, comparison between measurements and simulations. Both the spacer of $10 \mathrm{~mm}$ and 30 $\mathrm{mm}$ are considered (separation between the horn aperture and the polarizer).

edges of the polarizer is at least $10 \mathrm{~dB}$ lower than the level at its the center (considering the spacing of 10 and $30 \mathrm{~mm}$ and at both frequency bands). This criterion allows to assure that most of the energy is captured by the polarizer and very little radiation is reaching its edges or the support. Diffraction is thus avoided, as well as other possible sources of indirect radiation that would mask the polarizer influence in the pattern radiated by the horn.

The measured results are presented from Fig. 9 to Fig. 11. Two experiments (one with the spacer of $10 \mathrm{~mm}$ and another one with the spacer of $30 \mathrm{~mm}$ ) are always compared. In addition, these results are compared with representative simulations of the DUT. The support has been modelled as a dielectric material with $\epsilon_{r}=3$ and $\tan \delta=0.02$, values that emulate the photosensitive resin used to 3D-print the support. Figure 8 shows the realized gain of the two polarizations in the two frequency bands. Firstly, dual-band, opposite circular polarization conversion is achieved. The incident $45^{\circ}$ wave is transformed to RHCP in $10 \mathrm{GHz}$ and to LHCP in $15 \mathrm{GHz}$. The cross-polarization discrimination ranges from 15 to 20 $\mathrm{dB}$, which is in good agreement with simulated predictions. It should be noted that these values are worse than what was predicted for the FSS operation in Section III. This is explained by the wavefront created by the horn in the near field, that differs from the uniform plane wave ideally considered. Differences in the null of cross-polarization between simulations and measurements are mainly attributed to the alignment between polarizer and horn, which is done manually, and it is not perfectly $45^{\circ}$. For the sake of completeness, the DUT has been simulated without considering losses in the dielectric support. The differences observed in the gain computed at broadside with respect to the measurements remain always below 0.1 $\mathrm{dB}$. Figure 9 shows the radiation patterns at the centre of the two bands $(10 \mathrm{GHz}$ and $15.2 \mathrm{GHz})$. Again, good agreement is obtained between simulations and measurements, except for the cross-polarization in broadside and a cone of around $10^{\circ}$ around broadside. As in the previous result, differences are attributed to the alignment between horn and polarizer. Finally, the $S_{11}$ parameter of the DUT is shown in Fig. 11. Here, it is possible to see the filtering behaviour of the FSS, which induces transmission in the targeted bands and reflects the power back everywhere else. The return loss in the operation bands is higher than $8 \mathrm{~dB}$, with good agreement between

\section{ACCEPTED MANUSCRIPT}




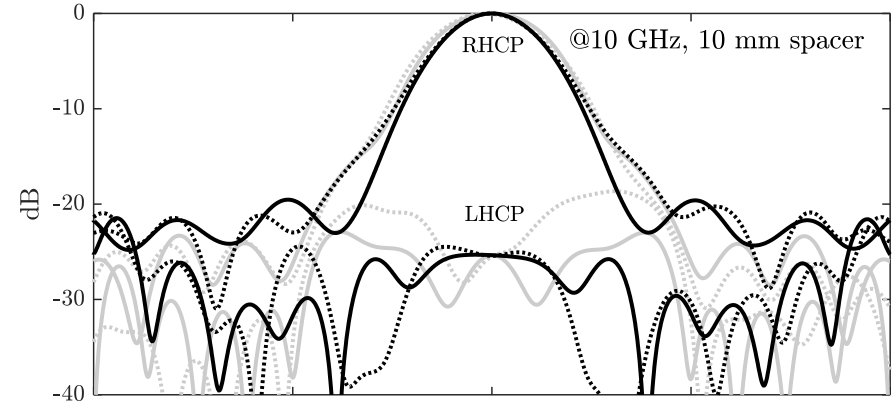

(a)

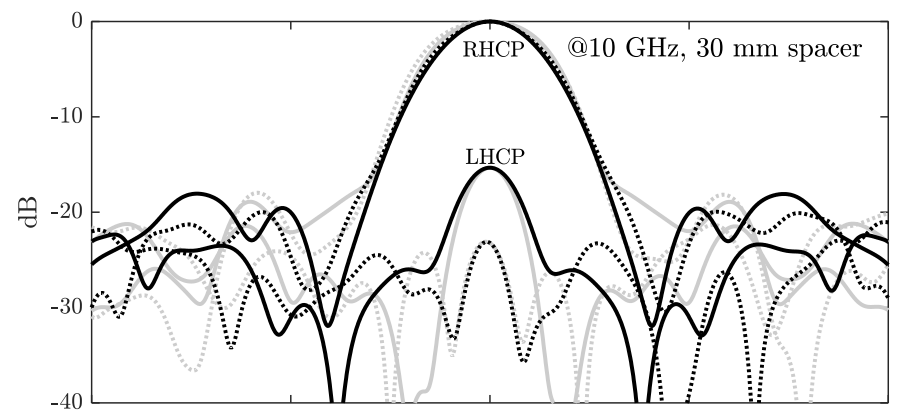

(b)

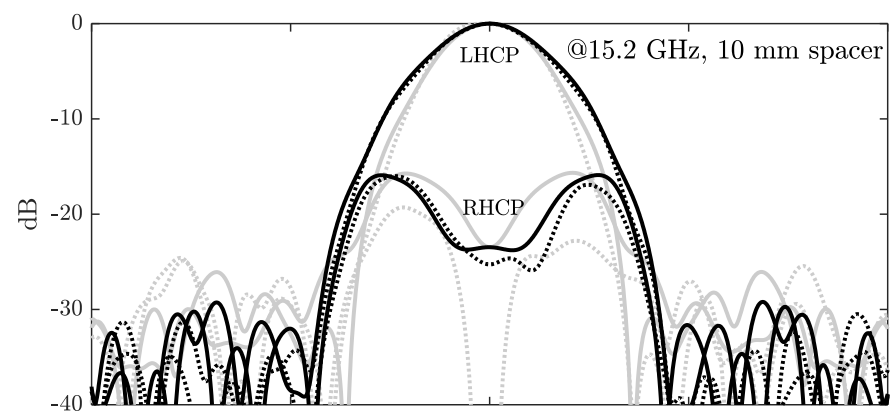

(c)

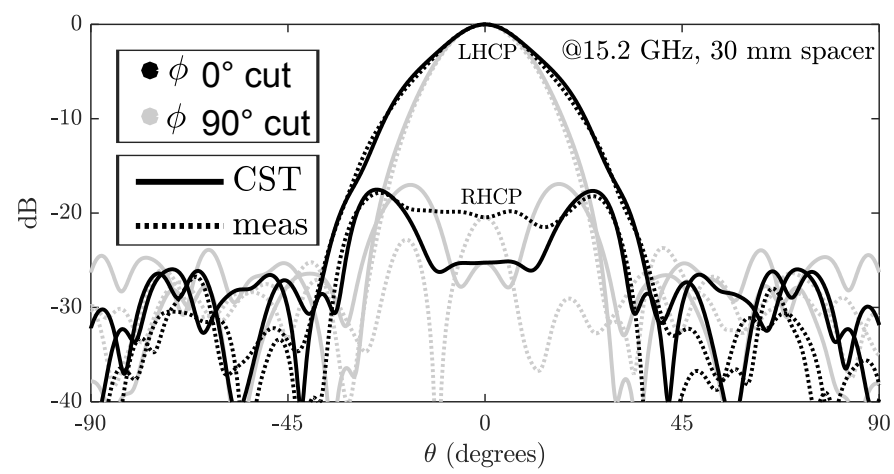

(d)

Fig. 10. Normalized radiation patterns, comparison between measurements and simulations. Results obtained at $10 \mathrm{GHz}$ and $15.2 \mathrm{GHz}$, considering a spacer of $10 \mathrm{~mm}$ and $30 \mathrm{~mm}$ (separation between the horn aperture and the polarizer) .

measurements and simulations.

\section{CONCLUSION}

A versatile 3-D periodic structure has been here presented which consists of a monolithic block of metal. The topology of the corresponding unit-cell is designed to provide dualpolarization and dual-band capabilities thanks to perforations

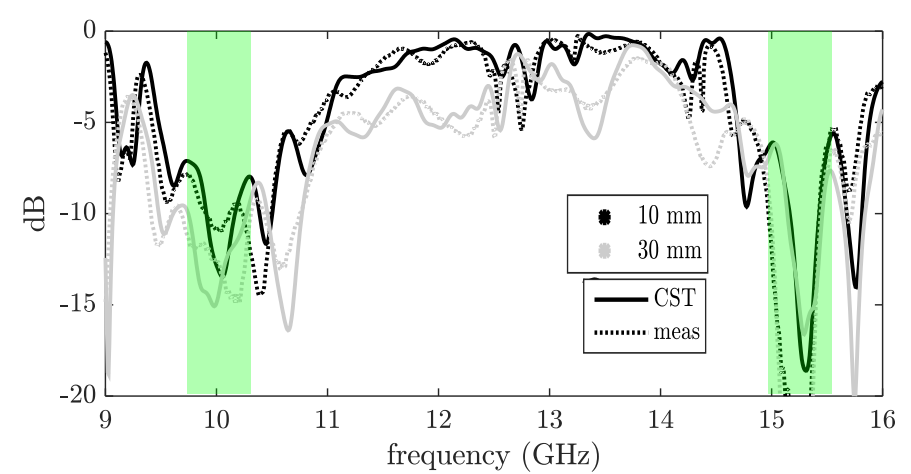

Fig. 11. $\mathrm{S}_{11}$ parameter of the prototype.

of different apertures in the lateral walls of a rectangular waveguide. An equivalent circuit is proposed to describe the scattering properties of such an structure. The versatility of the cell allows to synthesize frequency filtering responses and linear-to-circular polarization conversion. Three illustrative examples are proposed, including a dual-band polarizer inducing orthogonal sense of $\mathrm{CP}$ rotation at each band. The latter is manufactured and measured, showing very good performance and agreement with theoretical predictions.

\section{REFERENCES}

[1] A. K. Rashid, Z. Shen, and S. Aditya, "Wideband microwave absorber based on a two-dimensional periodic array of microstrip lines," IEEE Trans. Antennas Propag., vol. 58, no. 12, pp. 3913-3922, Dec. 2010.

[2] C. Pelletti, R. Mittra, and G. Bianconi, "Three-dimensional fss element with wide frequency and angular response," in 2012 International Symposium on Antennas and Propagation Society, Chicago, USA, July 2012.

[3] C. Molero and M. García-Vigueras, "Circuit modeling of 3-D cells to design versatile full-metal polarizers," IEEE Trans. Microw. Theory Techn., vol. 67, no. 4, pp. 1357-1369, March 2019.

[4] A. Lai and C. Caloz, "Composite right/left-handed transmission line metamaterials," IEEE Microw. Magazine, vol. 5, no. 3, pp. 34-50, Sept. 2004.

[5] J. B. Pendry, A. J. H. D. J. Robbins, and W. J. Stewart, "Magnetism from conductors and enhanced nonlinear phenomena," IEEE Trans. Microw. Theory Techn., vol. 47, no. 11, pp. 2075-2084, Nov. 2004.

[6] A. K. Rashid and Z. Shen, "Three-dimensional frequency selective surface," in 2010 International Conference on Communications, Chengdu, China, 2010.

[7] R. Kronberger and P. Soboll, "New 3D printed microwave metamaterial absorbers with conductive printing materials," in Proceedings of the $46^{\text {th }}$ European Microwave Conference (EuMC 2016), London, United Kingdom, Oct. 2016.

[8] A. K. Rashid and Z. Shen, "A novel band-reject frequency selective surface with pseudo-elliptic response," IEEE Trans. Antennas Propag., vol. 58, no. 4, pp. 1220-1226, April 2010.

[9] A. A. Omar and Z. Shen, "Multiband high-order bandstop 3-d frequencyselective structures," IEEE Trans. Antennas Propag., vol. 64, no. 6, pp. 2217-2226, June 2016.

[10] B. Sanz-Izquierdo and E. A. Parker, "3D printing of elements in frequency selective arrays," IEEE Trans. Antennas Propag., vol. 62, no. 12, pp. 6060-6066, Dec. 2014.

[11] J. Zhu, W. Tang, C. Wang, and C. Huang, "Dual-polarized bandpass frequency-selective surface with quasi-elliptic response based on square coaxial waveguide," IEEE Trans. Antennas Propag., vol. 66, no. 3, pp. 1331-1339, March 2018.

[12] C. Pelletti, G. Bianconi, R. Mittra, and Z. Shen, "Frequency selective surface with wideband quasi-elliptic bandpass response," Electronics Lett., vol. 49, no. 17, pp. 1052-1053, Aug. 2013.

[13] B. Liang and M. Bai, "Subwavelength three-dimensional frequency selective surface based on surface wave tunneling," Optics Express, vol. 24, no. 13, pp. 14697-14702, June 2016. 
[14] D. Z. Zhu, M. D. Gregory, P. L. Werner, and D. H. Werner, "Fabrication and characterization of multiband polarization independent 3-d printed frequency selective structures with ultrawide fields of view," IEEE Trans. Antennas Propag., vol. 66, no. 11, pp. 6096-6105, Nov. 2018.

[15] W. Wang, Q. Cao, and Y. Zheng, "Bandstop frequency selective structure based on stepped-impedance loop resonators: design, analysis and measurements," IEEE Trans. Antennas Propag., vol. 67, no. 2, pp. 1053 1064, Feb. 2019.

[16] X. Huang, C. Yang, Z. Lu, and P. Liu, "A novel frequency selective structure with quasi-elliptic bandpass response," IEEE Antennas Wireless Propag. Lett., vol. 11, pp. 1497-1500, 2012.

[17] Z. Lu, P. Guo, and X. Huang, "A novel three-dimensional frequency selective surface," IEEE Antennas Wireless Propag. Lett., vol. 11, pp. 588-591, 2012.

[18] W. Tang, J. Zhu, C. Wang, J. Ge, Z. Yu, and W. Zhuang, "Waveguide 3D fss by 3D-printing technique," in 2016 International Conference on Electromagnetics in Advance Applications (ICEAA), Cairns, Canada, 2016.

[19] A. Ericsson and D. Sjöberg, "Design and analysis of a multilayer meander line circular polarization selective structure," IEEE Trans. Antennas Propag., vol. 45.

[20] W. Tang, S. Mercader-Pellicer, G. Goussetis, H. Legay, and N. J. G. Fonseca, "Low-profile compact dual-band unit cell for polarizing surfaces operating in orthogonal polarizations," IEEE Trans. Antennas Propag., vol. 65 , pp. 1472 - 1477, March 2017.

[21] N. J. G. Fonseca and C. Mangenot, "High-performance electrically thin dual-band polarizing reflective surface for broadband satellite applications," IEEE Trans. Antennas Propag., vol. 64, pp. 640 - 649, Feb. 2016.

[22] M. L. N. Chen, L. J. Jian, W. E. I. Sha, W. C. H. Choy, and T. Itoh, "Polarization control by using anisotropic 3-d chiral structure," IEEE Trans. Antennas Propag., vol. 64, no. 11, pp. 4687-4694, Nov. 2016.

[23] K. X. Wang and H. Wong, "A wideband millimeter-wave circularly polarizer antenna with 3D printed polarizer," IEEE Trans. Antennas Propag., vol. 65, no. 3, pp. 1038-1046, March 2017.

[24] M. Silverinha, "Design of linear-to-circular polarization transformers made of long densely packed metallic helices," IEEE Trans. Antennas Propag., vol. 56, no. 2, pp. 390-401, Feb. 2008.

[25] M. García-Vigueras and C. Molero, "Circuital analysis of full-metal polarisers based on 3D unit-cells," in $48^{\text {th }}$ European Microwave Conference (EuMC 2018), Madrid, Spain, 2018.

[26] F. J. Vázquez-Sánchez, "Electromagnetic wave polarizer screen," EP 2 469653 Al, Jun. 2012.

[27] G. Collignon, "Polarisation device for a satellite telecommunications antenna and associated antenna," US Patent 0372820 A1, Dec. 2016.

[28] P. Naseri, S. A. Matos, J. R. Costa, C. A. Fernandes, and N. J. Fonseca, "Dual-band dual-linear-to-circular converter in transmission mode application to k/ka-band satellite communications," IEEE Trans. Antennas Propag., vol. 66, no. 12, pp. 7128-7137, Dec. 2018.

[29] M. Hosseini and S. V. Hum, "A systematic circuit-based approach to efficiently realize single- and dual-band circular polarizers," in Proc. 12th European Conf. Antennas Propag. (Eucap), London, United Kingdom, April 2018.

[30] H. B. Wang and Y. J. Cheng, "Single-layer dual-band linear-to-circular polarization converter with wide axial ratio bandwidth," IEEE Trans. Antennas Propag., vol. 67, pp. 4296 - 4301, 2019.

[31] P. Naseri and V. Hum, "A dual-band dual-circularly polarized reflectarray for $k / k a$-band applications," in Proc. 13th European Conf. Antennas Propag. (Eucap), Krakow, Poland, March 2019.

[32] N. Marcuvitz, Waveguide Handbook. New York, USA: McGraw-Hill Book Company, 1951.

[33] F. Costa, A. Monorchio, and G. Manara, "An overview of equivalent circuit modeling techniques of frequency selective surfaces and metasurfaces," Aces Journal, vol. 29, no. 12, pp. 960-973, 2014.

[34] J. Ge, J. Zhu, H. Zhang, , W. Zhuang, and W. Tang, "3-D square coaxial waveguide fss and equivalent circuit model," in 2016 Progress in Electromagnetic Research Symposium (PIERS 2016), Shanghai, China, 2016.

[35] J. F. Johansson, M. Viberg, J. Petersson, and P. Magnusson, "An all-metal k-band reflector antenna for a mechanically steerable data downlink system," in 2015 9th European Conference on Antennas and Propagation (EuCAP), April 2015, pp. 1-3.

[36] N. Chahat, B. Cook, H. Lim, and P. Estabrook, "All-metal dualfrequency rhcp high-gain antenna for a potential europa lander," IEEE Trans. Antennas Propag.,, vol. 66, no. 12, pp. 6791 - 6798, Dec. 2018.
[37] X. Fang, W. Wang, G. Huang, Q. Luo, and H. Zhang, "A wideband low-profile all-metal cavity slot antenna with filtering performance for space-borne sar applications," IEEE Antennas and Wireless Propagation Letters, vol. 18, no. 6, pp. 1278-1282, June 2019.

[38] F. Medina, F. Mesa, and R. Marqués, "Extraordinary transmission through arrays of electrically small holes from a circuit theory perspective," IEEE Trans. Microw. Theory Tech., vol. 56, no. 12, pp. 3108-3120, Dec. 2008.

[39] C. Molero, R. Rodríguez-Berral, F. Mesa, and F. Medina, "Dynamical equivalent circuit for 1-d periodic compound gratings," IEEE Trans. Microw. Theory Tech., vol. 64, pp. 1195 - 1208, April 2016.

[40] F. Medina, F. Mesa, and D. C. Skigin, "Extraordinary transmission through arrays of slits: A circuit theory model," IEEE Transactions on Microwave Theory and Techniques, vol. 58, no. 1, pp. 105-115, Jan 2010.

[41] H. Cao, X. Wu, Y. Pi, J. Liu, H. Xu, Z. Meng, Y. Yu, and J. Fan, "A novel chiral metamaterial circular polarizer based on e-shaped structure," in 2016 IEEE International Symposium on Antennas Propag. (APSURSI), Fajardo, Puerto Rico, July 2016.

[42] N. Nathrath, R. Gehring, A. Hoehn, M. Tebbe, M. Trumper, and H. Wolf, "The electroplated lightweight inter-satellite antenna (lisa) system for kaband with low-loss, dual polarization, waveguide rotary joint,", in $38^{\text {th }}$ Antenna workshop on Innovative Antenna systems and Technologies for Future Space Missions, Noordwijk, The Netherlands, October 2017.

[43] www.mvgworld.com/en/system/files/starlab2014.pdf.

[44] A. I. Dimitriadis, T. Debogovic, M. Favre, M. Billod, L. Barloggio, J. Ansermet, and E. de Rijk, "Polymer-based additive manufacturing of high-performance waveguide and antenna components," Proceedings of the IEEE, vol. 105, no. 4, pp. 668-676, April 2017.

[45] T. A. Milligan, Modern Antenna Design. Wiley-IEEE Press, 2005. 